\title{
Usefulness of lateral radiographs for detecting tuberculous lymphadenopathy in children - confirmation using sagittal CT reconstruction with multiplanar cross-referencing
}

\author{
Savvas Andronikou, MB ChB, FCRad, FRCR (Lond), PhD \\ Department of Radiology, Faculty of Health Sciences, University of the Witwatersrand, Johannesburg
}

Dirk Johannes van der Merwe, MB ChB

Department of Radiology, Stellenbosch University

\author{
Pierre Goussard, MMed (Paed) \\ Robert P Gie, FCP \\ Department of Paediatrics and Child Health, Stellenbosch University
}

Nicolette Tomazos, BA

Commerce Faculty, Department of Management Studies, University of Cape Town

Corresponding author: S Andronikou (docsav@mweb.co.za)

Background. Diagnosis of pulmonary tuberculosis (PTB) in children remains difficult. Lateral chest radiographs are frequently used to facilitate diagnosis, but interpretation is variable. In this study, lateral chest radiographs (CXRs) are evaluated against sagittal CT reconstructions for the detection of mediastinal lymphadenopathy.

Aim. To correlate suspected lymphadenopathy on lateral CXR with sagittal CT reconstructions and determine which anatomical group of lymph nodes contributes to each lateral CXR location.

Methods and materials. Thirty TB-positive children's lateral CXRs were retrospectively reviewed for presence of mediastinal lymphadenopathy in 3 pre-determined locations in relation to the carina: retrocarinal, subcarinal and precarinal. Findings of the CT sagittal reconstructions were then correlated with the CXRs for the presence of lymphadenopathy in the same 3 pre-determined areas across the width of the mediastinum. Axial and coronal CT crossreferencing confirmed the position of the lymphadenopathy.

Results. The most frequent locations for lymphadenopathy were the subcarinal (28) and right hilar (25). Sensitivity and specificity values of the CXRs were moderate, with the precarinal region having the best sensitivity and specificity for presence of lymphadenopathy. Contribution to each zonal group on lateral CXR were from multiple anatomical lymph node sites.

Conclusion. The precarinal zone on CXR had the best specificity and sensitivity, and represented mainly subcarinal and right hilar lymph node groups. Attention should be paid to this area on lateral CXRs for detecting lymphadenopathy in children with suspected PTB.

S Afr J Rad 2012;16(3):87-92. DOI:10.7196/SAJR.655
Diagnosis of pulmonary tuberculosis (PTB) in children remains a difficult problem, ${ }^{1,2}$ and radiography is frequently used for elucidation. ${ }^{1-3}$ Interpretation of chest radiographs (CXRs), however, is variable when measured against computed tomography (CT), which is considered the gold standard. ${ }^{4,5}$ In the present study, the lateral CXR - a readily available and inexpensive diagnostic tool - is evaluated against sagittal CT reconstructions for the detection of mediastinal and hilar lymphadenopathy. Densities suspected of representing lymphadenopathy on lateral CXR have not been confirmed using CT previously, and the contribution of different lymph node groups to the lateral CXR locations remain unproven. Sagittal CT reconstructions were used for comparison with lateral radiographs for determining the position of suspected TB lymphadenopathy. Multiplanar cross-referencing was used to confirm the presence and location of lymphadenopathy. The sensitivity and specificity of lateral CXR locations for the presence of lymphadenopathy was determined against the CT gold standard.

\section{Aim}

We set out to compare detection of tuberculous lymphadenopathy on lateral CXRs with sagittal CT reconstructions and determine which anatomical group of lymph nodes contributes to locations on lateral CXR by using multiplanar cross-referencing.

\section{Methods and materials}

A retrospective descriptive study was undertaken. Children $<13$ years of age were included when a diagnosis of $\mathrm{TB}$ was made by positive culture of bronchoscopic washings or gastric aspirates and when CXRs (PA and lateral) as well as a chest CT were available (CT scans were performed for the indication of airway compression, as not all children with TB undergo CT routinely). Thirty children meeting the 


\section{ORIGINAL ARTICLE}

criteria were included, working retrospectively and consecutively from the start date. Patients were excluded when there was severe airspace disease (i.e. whiteout) and very poor-quality radiographs. Where a case failed to meet the study criteria, the next sequential case was selected.

The chest CTs were retrieved from our electronic database, and multiplanar reconstructions (MPRs) were performed retrospectively using Voxar 3D 4.2 (Barco, Belgium). Lateral CXRs were read from hard copy. Reading of plain radiographs and CT was performed by a paediatric radiologist (SA) with a special interest in TB. Plain radiographs were read first; CTs were read at a different sitting with the reader blinded to patient details, history and previous radiological findings.

Lateral CXRs were read for presence of lymphadenopathy in 3 predetermined locations as 'negative' or 'positive'. Location positions were determined by extending lines from the anterior and posterior walls of the trachea beyond the carina and drawing another line horizontally (perpendicularly) to these below the level of the right upper lobe bronchus. The horizontal line was drawn at this level to exclude densities representing the major vascular structures around the upper regions of the hila (right and left main pulmonary arteries and aortic arch which are indistinguishable from lymph node soft tissue densities). Three areas were thus viewed in relation to the carina: retrocarinal (posterior to the lines drawn), subcarinal (between the lines drawn) and precarinal (anterior to the lines drawn) to represent actual lymph node

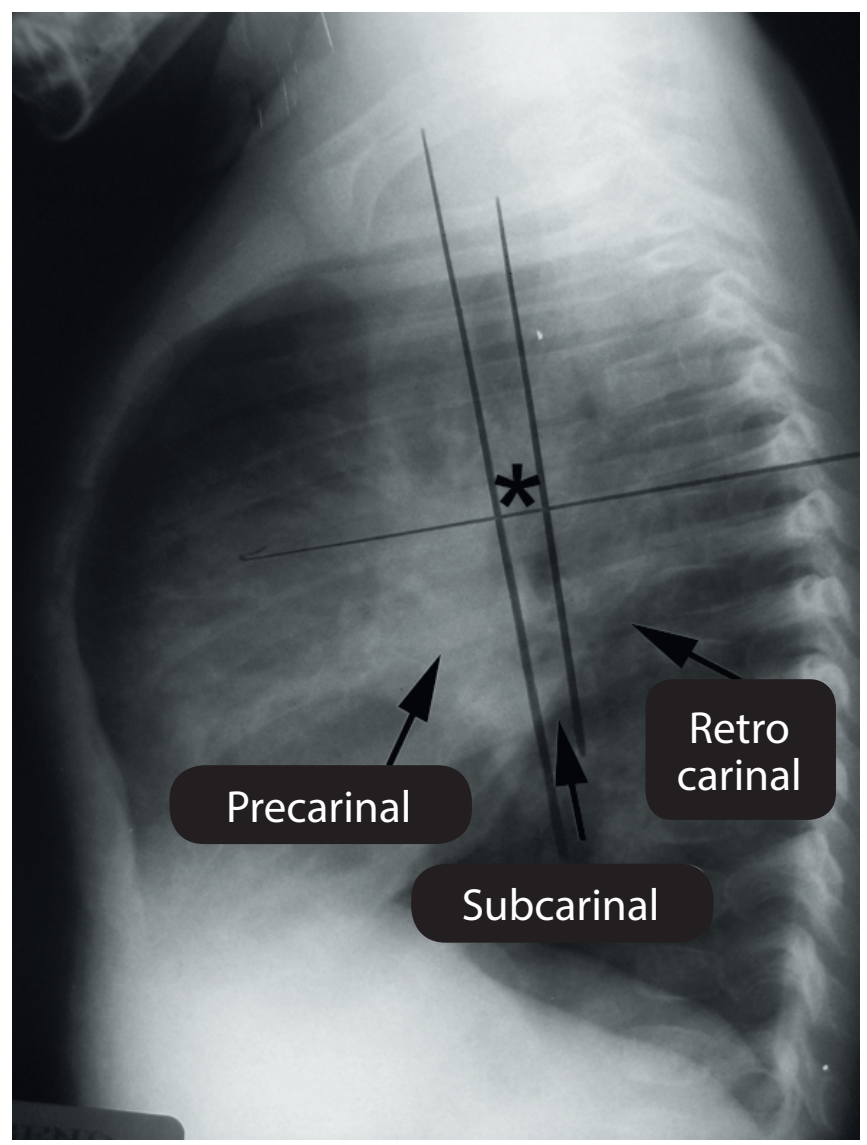

Fig. 1. Lines were drawn along the anterior and posterior tracheal margins as well as a horizontal (perpendicular) line below the right upper lobe bronchus (asterisk). Three zones were thus created below the horizontal line: precarinal, subcarinal and retrocarinal. Density can be seen in all three zones in this example, suggesting lymphadenopathy.

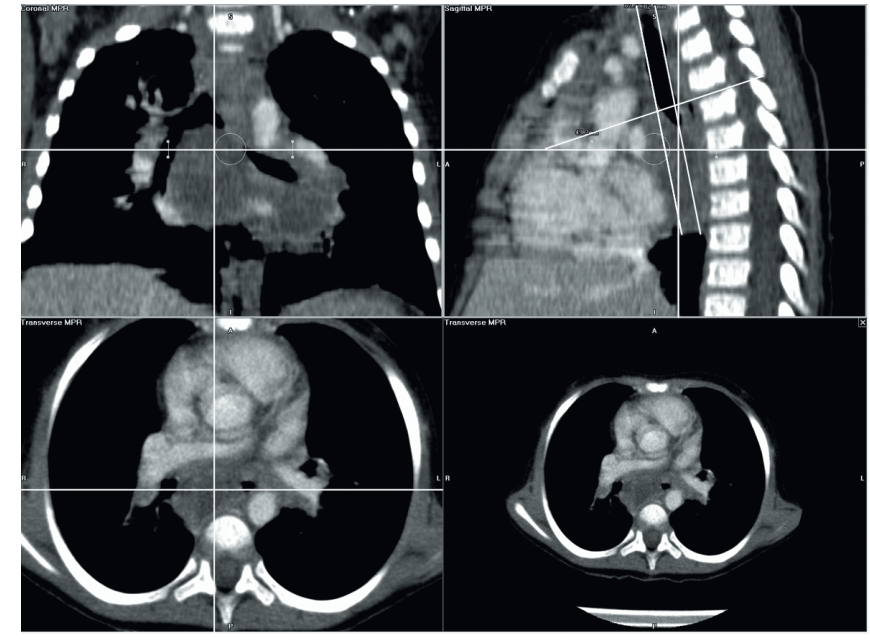

Fig. 2. The same three lines were digitally imposed on the sagittal MPR CT (top right image). The cross-hair in the coronal and axial planes indicates the subcarinal zone, and the soft-tissue density corresponds to a large subcarinal lymph node group.

group locations (Fig. 1). Lymphadenopathy in the 3 zones was reported when conglomerate oval densities were noted. Densities were reported to be vascular if these were linear and diverging from the central point. Airspace disease was reported when confluent densities containing air bronchograms were present.

Sagittal MPRs were then read for the presence of lymphadenopathy. The same 3 lines that were drawn on the lateral CXR were digitally superimposed on the mid- and para-sagittal CT reconstructions. The sagittal MPR scan was then read for the presence of lymphadenopathy in the same 3 predetermined areas as a surrogate for lateral CXR, in the midline and every para-sagittal position across the mediastinum. Position of adenopathy was confirmed on axial and coronal plane CT using the cross-referencing cross-hair (Fig. 2). No size dimension for pathological mediastinal nodes was used and the presence of any lymph nodes was recorded as abnormal.

Zonal findings on lateral CXR and sagittal MPR were then correlated. Sensitivity, specificity, positive predictive value and negative predictive value were determined overall and for each of the predetermined positions using the CT images as the gold standard. Ethical approval for this study was obtained from the local ethics board; the study was conducted in conformance with the guiding principles of the Declaration of Helsinki.

\section{Results}

The 30 children in this study ( 15 male and 15 female) ranged from 0 - 7 years, with a mean age of 2 years. Table 1 shows how lymphadenopathy was distributed as seen in the predetermined zones on lateral CXR and sagittal MPR. Anatomical locations for all lymph nodes seen on (axial and MPR) CT were in descending order of frequency: subcarinal (28), right hilar (25), right para-tracheal and azygo-oesophageal (22), precarinal (21) and left hilar (15).

Mismatches in diagnosis of the presence or absence of lymph nodes are summarised in Table 2. Sensitivity and specificity values of CXR (taking CT as the gold standard) were moderate, with the precarinal region having the best sensitivity and specificity for the presence of lymphadenopathy. Positive predictive values were high at all zones, as was the incidence of lymphadenopathy in the chosen population; Table 


\section{ORIGINAL ARTICLE}

Table 1. Positive findings of lymphadenopathy on lateral CXR and sagittal CT reconstruction according to zone in 30 children with PTB

\begin{tabular}{llll}
\hline Positive for adenopathy & Lateral CXR & Sagittal CT & Difference \\
\hline Retrocarinal & 22 & 25 & 3 \\
Subcarinal & 22 & 28 & 6 \\
Precarinal & 20 & 26 & 6
\end{tabular}

Table 2. Correlation of lateral CXR and sagittal CT reconstruction according to zone in 30 children with PTB

\begin{tabular}{llll}
\hline Zone & Positive on CXR and CT & Negative on CXR and CT & Mismatch \\
\hline Retrocarinal & 19 & 2 & 9 \\
Subcarinal & 21 & 1 & 8 \\
Precarinal & 19 & 3 & 8
\end{tabular}

Table 3. Sensitivity, specificity, PPV and NPV of lateral CXR for presence of lymphadenopathy when compared with sagittal CT reconstructions by zone

\begin{tabular}{lllll}
\hline & Retrocarinal & Subcarinal & Precarinal & Overall \\
\hline Sensitivity & $76 \%$ & $75 \%$ & $73 \%$ & $75 \%$ \\
Specificity & $40 \%$ & $50 \%$ & $75 \%$ & $55 \%$ \\
PPV & $86 \%$ & $95 \%$ & $95 \%$ & $92 \%$ \\
NPV & $25 \%$ & $12.5 \%$ & $30 \%$ & $23 \%$ \\
PPV = positive predictive value; NPV = negative predictive value. & & & & \\
\end{tabular}

Table 4. Contribution of lymph node groups to defined zones on sagittal CT (and by inference on lateral CXR) using only true positives (see Table 3)

\begin{tabular}{llllll}
\hline Zone & $\begin{array}{l}\text { Positive on sagittal CT } \\
\text { and lateral CXR }\end{array}$ & Right hilar & Left hilar & Subcarinal & $\begin{array}{c}\text { Azygo- } \\
\text { oesophageal }\end{array}$ \\
\hline Retrocarinal & 19 & 8 & 7 & 9 & 0 \\
Subcarinal & 21 & 17 & 7 & 21 & 15 \\
Precarinal & 19 & 16 & 8 & 15 & 12 \\
\end{tabular}

3 provides a summary.

Contributions of the different lymph node groups to each of the zonal groups on the lateral CXR (taking only true positives into account) show a predominant contribution to the retrocarinal zone from azygooesophageal nodes, subcarinal zone from subcarinal nodes, and the precarinal zone from right hilar and subcarinal nodes. A more detailed summary of this correlation is in Table 4.

\section{Discussion}

TB remains a significant cause of mortality and morbidity in South Africa and worldwide. The Western Cape in South Africa had an incidence of 468 cases per 100000 people in 1998, which is among the highest in the world. ${ }^{6} \mathrm{HIV}$ has further increased the incidence of TB. Diagnosing the disease in children is a challenge, as $56-65 \%$ of children are asymptomatic at the time of diagnosis. The Mantoux test reaction lags behind radiological findings. ${ }^{1-3,7}$ Sputum is frequently unavailable, and gastric washings are positive in only $30-40 \%$ of cases. ${ }^{3}$ Because of all these factors, Mycobacterium tuberculosis is isolated in less than $50 \%$ of paediatric cases. The diagnosis of PTB therefore often relies on imaging findings.

Children from birth to 3 years have a high prevalence of lymphadenopathy (92 - 96\%) and a lower prevalence of parenchymal abnormalities, and this is the radiological hallmark of primary TB. ${ }^{2,3,6}$ In almost half of early childhood cases, lymphadenopathy is the sole radiological manifestation. ${ }^{3}$ Because of these factors, strong reliance is placed on CXR in aiding the diagnosis through detection of mediastinal adenopathy. As the WHO's proposed criteria for PTB include a suggestive appearance on $\mathrm{CXR},{ }^{8}$ children diagnosed with PTB through radiography are treated with a full course of anti-tuberculous drugs. ${ }^{9}$

The lateral CXR is still frequently used in South Africa, as it is thought to assist with the detection of hilar and mediastinal lymph nodes. ${ }^{10}$ It demonstrates lymphadenopathy as lobulated densities appearing in the lower half of a doughnut configuration around the carina. The upper half of the doughnut is made up of the aortic arch 


\section{ORIGINAL ARTICLE}

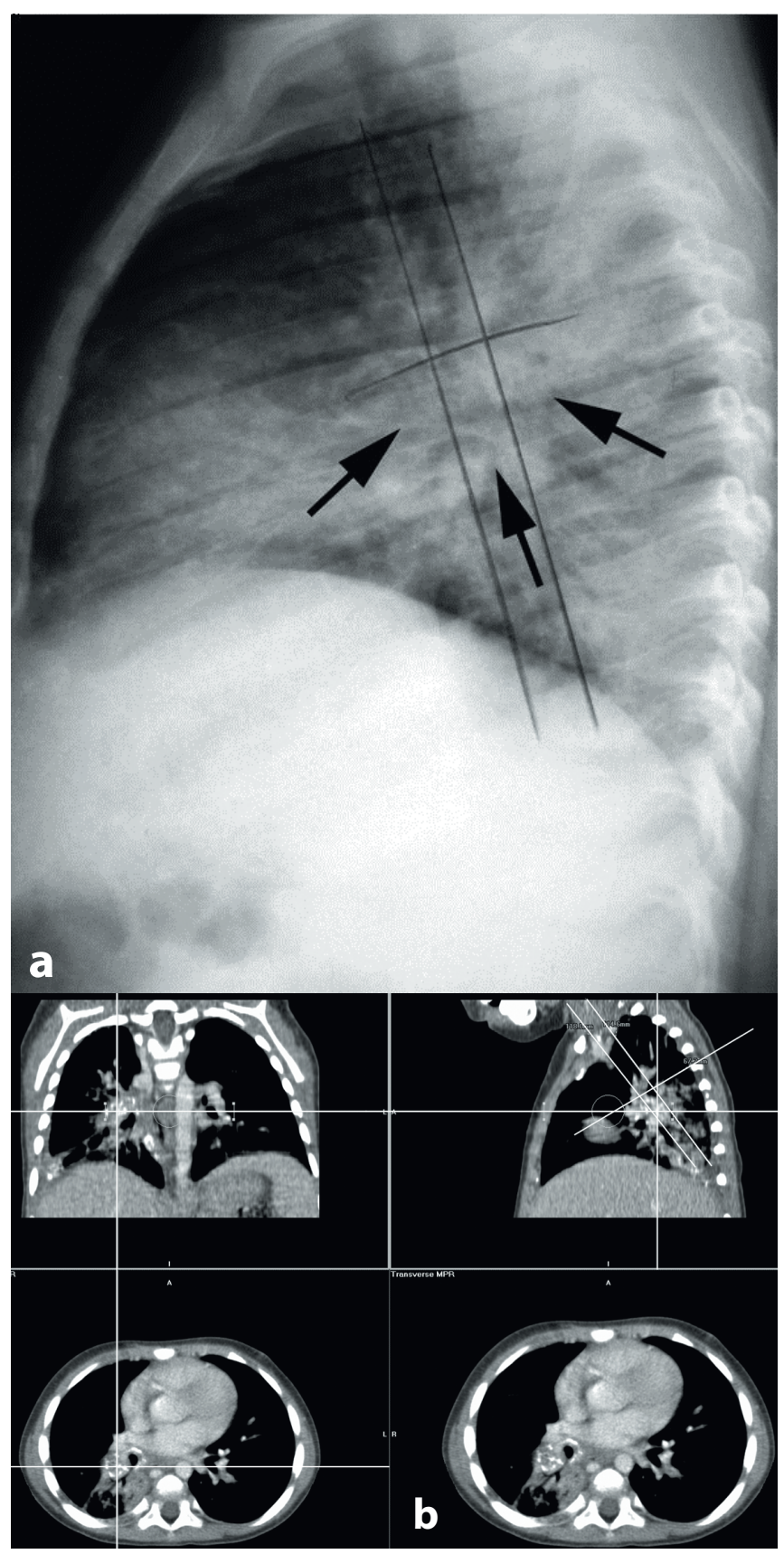

Figs $3 a$ and b. Lateral CXR (a) in this 2-year-old boy demonstrates densities in all 3 predetermined zones (arrows). The MPR CT (b) confirms the lateral CXR findings, and the cross hair position demonstrates that a calcified right hilar node is contributing to density seen in the subcarinal zone.

and the main pulmonary arteries, ${ }^{11,12}$ which is the reason why we have defined the region below the right upper lobe bronchus for evaluation in our methodology.

Worldwide use of the lateral CXR is still a contentious issue. Some feel that it adds value to frontal projections, which are frequently confusing owing to overlapping anatomical structures. ${ }^{13}$ In the presence of other pathology (e.g. infiltrates and miliary TB), the lateral projection was found to be more accurate in detecting hilar lymphadenopathy by Smuts et al. Their study confirmed the value of the lateral CXR in paediatric PTB but found difficulty in differentiating left and right hilar lympadenopathy on frontal CXR, as the heart obscures the left hilum. ${ }^{14}$

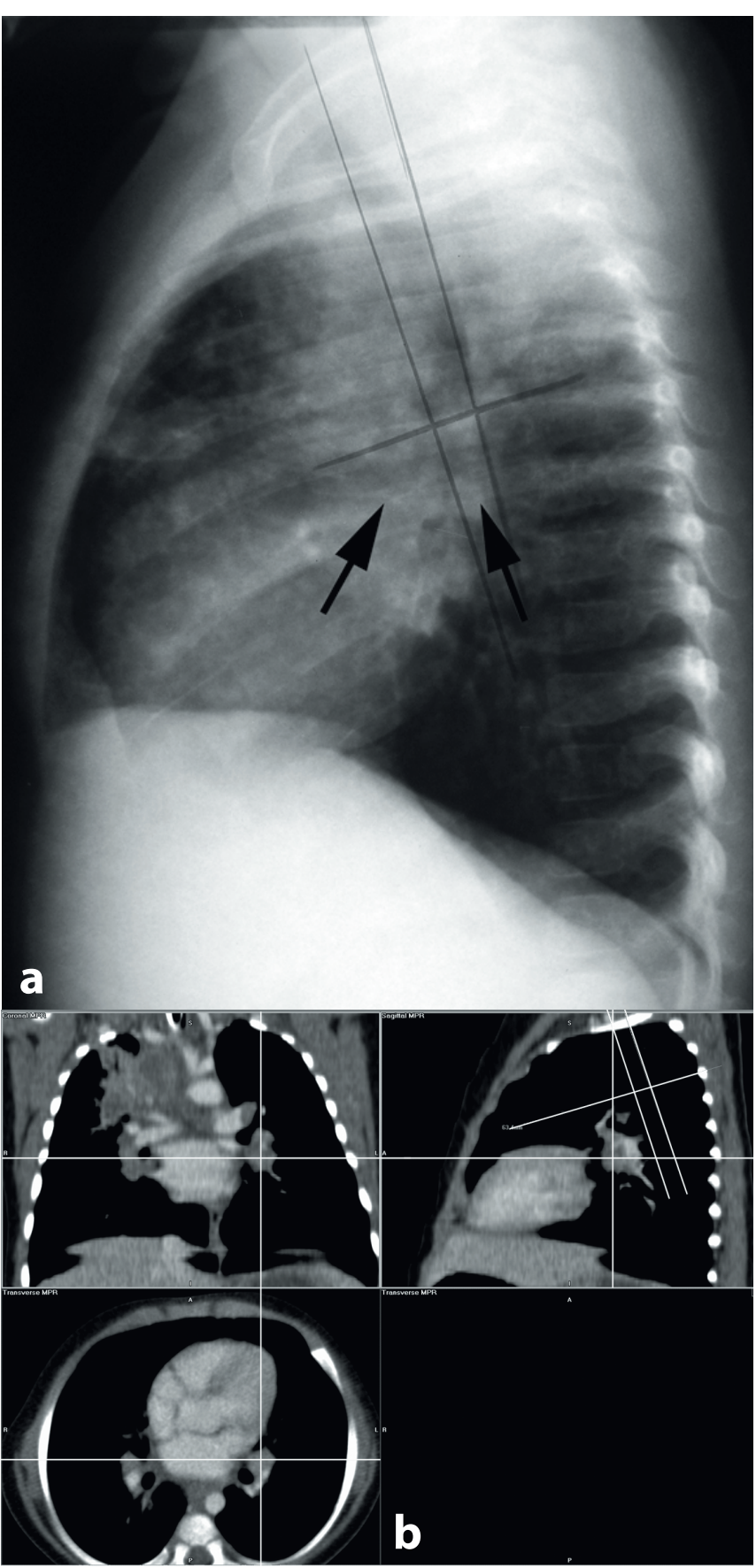

Figs $4 a$ and $b$. In this 1-year-old girl, the lateral CXR (a) demonstrates oval density in the precarinal and subcarinal zones (arrows). The MPR CT (b) cross-hair position confirms that there is a left hilar node that is contributing to density in the precarinal zone. (The MPR in this patient also revealed adenopathy in the retrocarinal zone that was missed on lateral CXR.) Note how the lines drawn along the trachea are projected into the parasagittal position to reflect the corresponding lateral CXR zones.

Swingler et al. found low accuracy among expert observers in detecting adenopathy on plain film. Their study found that the lateral CXR adds little extra value, but that diagnostic accuracy could be improved by refining radiological criteria for lymphadenopathy. ${ }^{9}$ Such criteria have not yet been determined.

TB lymphadenopathy occurs predominantly in the subcarinal and right hilar areas. ${ }^{3,4,15}$ Hila are affected more often than the paratracheal 
region since lymph drainage occurs from the hilum to paratracheal nodes. ${ }^{14}$ Glazer et al. found the largest mediastinal lymph nodes in normal individuals also occur in the subcarinal and right tracheobronchial areas. ${ }^{16}$ These features are useful when trying to determine diagnostic criteria on radiographs.

CT is considered the gold standard for the detection of lymphadenopathy, while CXR has been shown to be insensitive for lymphadenopathy detection. ${ }^{4,5}$ However, in many areas of South Africa and the developing world, CT is not readily available. Cost constraints and the high radiation dose of CT limit routine use of this modality in diagnosing PTB. It is therefore best to use CT to define more sensitive and specific CXR criteria.

The size of pathological lymph nodes in PTB on CT is also not well determined. ${ }^{17}$ Some studies adopt the notion that any visible mediastinal and hilar lymph nodes on CT are abnormal in children. Newer scanners, however, detect smaller nodes, which creates high sensitivity but lower specificity as some visualised nodes may only reflect reactive changes. ${ }^{6}$ Diagnosing nodes of TB specifically may be more accurate on CT owing to the typical post-contrast enhancement patterns. ${ }^{6,15,18,19}$ Calcification helps in lymph node detection (Fig. 3) but is present in only $15-21 \%$ of children with PTB, and not before 6 months of age. ${ }^{6}$

A previous study done Hammersley et al. showed high correlation between subcarinal density on PA films and subcarinal lymphadenopathy on $\mathrm{CT}^{20}{ }^{20}$ No studies to date correlating findings of lymphadenopathy on lateral CXR with those on CT could be found.

In our study, we chose a group with a (pre-selection bias) high incidence of lymphadenopathy, as the goal was not to demonstrate an incidence of lymphadenopathy but rather to confirm the true site of origin of suspected lymphadenopathy on lateral CXR. Numerous locations of nodes are a common finding in primary $\mathrm{TB}$, and multiple groups contributed to each positive zone on lateral CXR. Predominant groups in certain zones follow the anatomical distribution of nodes. Examples are the azygo-oesophageal nodes in the retrocarinal zone. However logical this may seem, it has not been proven to date for lateral CXR imaging. Even though there was a high incidence of nodes, there was still significant mismatch between lateral CXR and MPR. Sensitivity in all zones was only moderately high, considering the high incidence and multiple sites of lymph nodes involved. Specificity for most zones was poor. Overall, the most frequent sites for lymphadenopathy in this study were subcarinal and right hilar and these two lymph node groups presented predominantly in the subcarinal and precarinal zones of the lateral CXR. The precarinal location on lateral CXR (Fig. 4) was the only group with high sensitivity and specificity for detecting lymph nodes when correlated with MPR.

\section{Limitations}

The population chosen had a very high incidence of lymph nodes. No normal control cases were used. Therefore, PPV and NPV might not reflect the situation in a regular population group. However, ethical considerations relating to high radiation exposure of chest CT in children prevent us from generating a control group. Use of CT scans for pathology other than TB as controls is also unwise in our population, as $\mathrm{TB}$ is endemic with the highest per capita incidence in the world.

\section{Conclusion}

This study proves only moderate correlation between findings of lymphadenopathy on lateral CXR and CT. The precarinal zone on CXR had the best specificity and sensitivity. In addition, this zone was contributed to by all the lymph node groups, and in particular by the subcarinal and right hilar groups, which are most frequently involved in primary TB. Most attention should be paid to the area anterior to a line extended from the anterior tracheal wall and below the right upper lobe bronchus when assessing the CXR for lymphadenopathy in children with suspected PTB.

The lateral CXR is still used in South Africa and many other parts of the world in assessing lympadenopathy in PTB in children, and research should aim to assist clinicians to focus on the areas of lateral CXR that have higher yield for the presence of lymphadenopathy.

1. Starke JR, Taylor-Watts KT. Tuberculosis in the pediatric population of Houston, Texas. Pediatrics 1989;84:28-35.

2. Weber AL, Bird KT, Janower ML. Primary tuberculosis in childhood with particular emphasis on changes affecting the tracheobronchial tree. Am J Roentgenol 1968;103:123-132.

3. Leung AN, Muller NL, Pineda PR, Fitzgerald JM. Primary tuberculosis in childhood: Radiographic manifestations. Radiology 1992;182:87-91.

4. Andronikou S, Brauer B, Galpin J, et al. Interobserver variability in the detection of mediastinal and hilar nodes on CT in children with suspected pulmonary tuberculosis. Pediatr Radiol 2005;35:425-428.

5. Delacourt C, Mani TM, Bonnerot V, et al. Computed tomography with a normal chest radiograph in tuberculous infection. Arch Dis Child 1993;69:430-432

6. Andronikou S, Joseph E, Lucas S, et al. CT scanning for the detection of tuberculous mediastinal and hilar lymphadenopathy in children. Pediatr Radiol 2004;34:232-236.

7. Salazar GE, Schmitz TL, Cama R, et al. Pulmonary tuberculosis in children in a developing country. Pediartics 2001;108:448-453.

8. World Health Organization. The worldwide magnitude of protein-energy malnutrition: An overview from the WHO. Provisional guidelines for the diagnosis and classification of the EPI target diseases for primary health care, surveillance and special studies, EPI/GEN/83/4. Geneva: World Health Organization, 1983.

9. Swingler GH, Du Toit G, Andronikou S, Van der Merwe L, Zar HJ. Diagnostic accuracy of chest radiography in detecting mediastinal lymphadenopathy in suspected pulmonary tuberculosis. Arch Dis Child 2005;90:1153-1156.

10. Cremin BJ, Jamieson DH. Childhood tuberculosis: modern imaging and clinical concepts. Berlin, New York: Springer Verlag, 1995.

11. Kuhn JP, Slovis TL, Silverman FN, et al. Mediastinum. In: Silverman FN, Kuhn JP, eds. Caffey’s Pediatric X-ray Diagnosis, 9th ed. St. Louis: Mosby; 1993:666-671.

12. Andronikou S, Wieselthaler N. Modern imaging of tuberculosis in children: thoracic, central nervous system and abdominal tuberculosis. Pediatr Radiol 2004;34:861-875.

13. Caffey J. Pediatric X-ray Diagnosis. Chicago: Year Book, 1978:479-480.

14. Smuts NA, Beyers N, Gie R P, et al. Value of the lateral chest radiograph in tuberculosis in children. Pediatr Radiol 1994;24:478-480.

15. Kim WS, Choi J, Cheon J, et al. Pulmonary tuberculosis in infants:Radiographic and CT findings. Am J Roentgenol 2006;187:1024-1033.

16. Glazer GM, Gross BH, Quint LE, et al. Normal mediastinal lymph nodes: Number and size according to American Thoracic Society Mapping. Am J Roentgenol 1985;144:261-265.

17. Andronikou S. Pathological correlation of CT-detected mediastinal lymphadenopathy in children: the lack of size threshold criteria for abnormality. Pediatr Radiol 2002;32:912.

18. Kim WS, Moon WK, Kim I, et al. Pulmonary tuberculosis in children: Evaluation with CT. Am J Roentgenol 1997;168:1005-1009.

19. Moon WK, Im J, Yeon KM, Han MC. Mediastinal tuberculous lymphadenitis:CT findings of active and inactive disease. Am J Roentgenol 1998;170:715-718.

20. Hammersley JR, Grum CM, Green RA. The correlation of subcarinal density visualized on plain chest roentgenograms with computed tomographic scans. Chest 1990;97:869-872. 